\title{
Does mechanical locking-base ceramic brackets reduce cracks at debonding?
}

\author{
Mohammad Hossein Ahangar Atashi, Mojgan Kachoei
}

DDS, MSD. Associate professor.Tabriz Faculty of Dentistry. Tabriz University of Medical Sciences, Iran.

Correspondence:

Orthodontic Department,

Faculty of Dentistry,

Daneshgah St. Azadi St. ,

Tabriz, IRAN

E-mail address: atashim_2006@yahoo.com

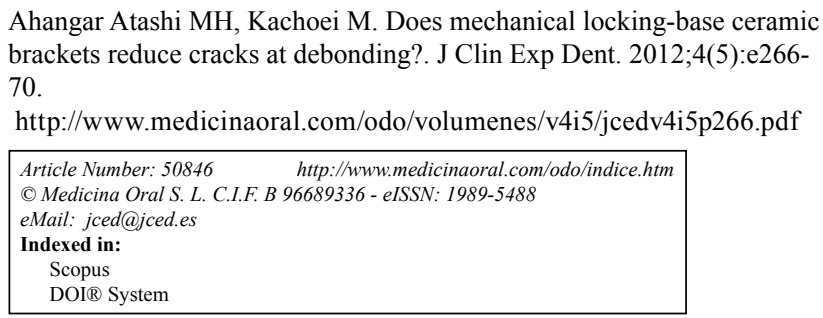

\begin{abstract}
Objectives: Debonding of chemically bonded ceramic brackets has been mostly led to enamel damages. This study aimed to evaluate enamel cracks followed by debonding ceramic brackets with mechanical retention.

Study Design: Sixty extracted human premolar teeth were randomly divided into two groups. Metallic brackets and recently available ceramic brackets with mechanical retention were bonded to the teeth in first (MB) and second (CB) group respectively. After a week, the brackets were debonded using a pair of bracket-removing pliers assembled to a testing machine. Optical stereomicroscope and trans-illumination techniques were used to calculate the cracks and measure their length before bonding and after debonding. Paired t-test, independent t-test and McNemar's test were used for statistical analysis.

Results: Metallic brackets showed higher mechanical bond strength compared to ceramic brackets with mechanical locks. In both groups, higher numbers of teeth with enamel cracks and a significant increase in the length of enamel cracks were evident subsequent to debonding. Inter-group deference in the number of teeth with cracks and the length of enamel cracks before or after debonding was not significant.

Conclusions: The risk of producing enamel cracks after debonding of ceramic brackets with mechanical retention is the same as metallic brackets.
\end{abstract}

Key word: enamel cracks, debonding, ceramic brackets. 


\section{Introduction}

Cracks, occurring as split lines in the enamel, are prone to debris and stains leading to discoloration of teeth and esthetic problems for the patients $(1,2)$.

Cracks on enamel are common but often are overlooked at clinical examination. Kracks can be distinguished by finger shad-owing in good light or, preferably, fiber-optic frans-illumination. Recently developed magnetic resonance imaging (MRI) technique, called SWeep Imaging with Fourier Transform (SWIFT), is capable to visualize dental tissues including enamel cracks (3).

Sometimes a sharp sound heard on debonding indicates the creation of pracks. Distinct horizontal and oblique cracks after debonding, particularly on teeth lother than maxillary canines and central incisors, have been blamed to debonding (4).

According to some studies bonding strength is a factor in producing enamel cracks. It has been demonstrated that the higher bonding strength of the adhesive is, the more likely the breakage shifts towards the enamel would be. The lack of ductility may generate stress in the adhesiveenamel interface which may produce enamel cracks at debonding $(5,6)$.

Stainless steel metallic brackets have been widely used among clinicians. Recently the need for esthetic appearance during treatment, especially for adults, has encouraged the use of ceramic brackets instead of metallic brackets $(2,7,8)$.

With chemically bonded ceramic brackets, the risk for creating enamel cracks is greater than for steel brackets because of their higher force needed to debond. More recent ceramic brackets with a mechanical lock in the base are available for bonding purposes. In this kind of brackets there is a vertical slot that will split the bracket by squeezing. Separation is at the bracket-adhesive interface. A few studies available on these newly designed ceramic brackets have emphasized that these cause fewer problems in debonding than do those using chemical retention $(2,8,9)$.

Mode of debonding has been another factor potentially capable of creating enamel cracks $(10,11)$. In the literature, most findings on bonding or debonding strength were based on laboratory studies that used one-way system force design using test machine and enamel damages after debonding ceramic brackets (with chemical bonding) were compared to steel brackets(with mechanical bonding).

This study aimed to compare ceramic brackets with mechanical locks to conventional steel brackets with regard to ability of producing enamel cracks at the time of debonding. In this study the original method of debonding with a twin-beaked pliers advocated by Bishara et al (5) was used to simulate clinical situation.

\section{Material and Methods}

This study was conducted in Tabriz University of Medi- cal Sciences, Iran. The protocol of the study was approved by regional medical research ethical committee. The materials of the study consisted of 60 upper premolar teeth (first or second) extracted for orthodontic treatment in a private clinic in Tabriz, Iran. The sample size formula, was used to estimate the size of study population in which $\alpha=5 \%$, power $=80 \%, d=0.6$ and $P=0.5$. All samples had equal mesio-distal size of $7.5 \pm 0.5 \mathrm{~mm}$ and no fillings, caries or structural anomalies were detected in macroscopic vision. None of the samples underwent chemical enamel processing e.g. $\left(\mathrm{H}_{2} \mathrm{O}_{2}\right)$ or bonding. After extraction, the samples were kept in $0.1 \%$ Thymol for 48 hours to prevent bacterial growth and dehydration, then they were stabilized in wax sheets in a way that their buccal surfaces remained exposed (12). The samples were randomly allocated into two groups of 30 : Ceramic Bracket group (CB) in which the teeth were bonded with ceramic brackets with mechanical retentive base (GAC International, ROTH 022 Inc, Allure III) and metallic bracket group (MB) in which the teeth were bonded with conventional stainless steel brackets (GAC international, ROTH 022 Inch, Ovation). All brackets' bases in two groups had rectangular form and were in the same size of $12 \mathrm{~mm}(2,13,14)$.

- Bonding: After elimination of debris and polishing with fluoride-free pumice, buccal surface of the samples, in area equal with the bracket bases, was etched by $37 \%$ phosphoric acid (3M, Dental Product, and St. Paul Mn 55144 ) for $15 \mathrm{~s}$ then were rinsed by water spray for $15 \mathrm{~s}$ and dried for $10 \mathrm{~s}$ from a distance of $2 \mathrm{~cm}$ so that the white etched area was observed. Transbond primer (3M/Unitek) and adhesive (transbond XT (3M/Unitek) were put on the bracket bases. A scalar was used to position the brackets on the midpoint of the buccal surface mesio-distaly and occluso-gingivaly. One point contact force was applied to the center of the brackets by the scalar so a tight fit of brackets to the teeth was achieved (15-16). Adhesive excesses around the brackets were removed by the sharp scalar. Bonding was preceded using a light curing unit (Astralis 7Ivoclar, Vivadent) with probe diameter of $8 \mathrm{~mm}$ and tedious intensity of $400 \mathrm{~mW} / \mathrm{cm}^{2}$. The brackets were irradiated for 20 seconds ( 10 seconds from mesial and 10 second from distal) by a distance of $2 \mathrm{~mm}$ (17). After bonding, the teeth were put in the incubator at $37^{\circ}$ of distilled water for 24 hours then they underwent thermocycling with 1000 cycles /min with temperature between $5^{\circ} \mathrm{C}$ and $55^{\circ} \mathrm{C}(5,17)$.

- Debonding: In this study the original method of debonding with a twin-beaked pliers advocated by Bishara was used to simulate clinical situation (5).

After a week, all brackets were debonded using a piece of double knife-edged bracket removing pliers (ODG 345 Invecta, GAC Corp. USA). Debonding force on the pick of pliers was measured by universal testing machine (Hunsfield Test Equipment, H5K-S model, Salsfords, 
Redhill, Surrey, England) to which was assembled. A custom-made jig held the pliers on the testing machine. A vertically oriented force system was designed using two opposite rods with $0.5 \mathrm{~mm}^{2}$ of bearing area and two hinges between rods and metallic bar (Fig. 1). A compressive force by the speed of $0.5 \mathrm{~mm}$ per minute was applied into the interface between bracket and tooth mesiodistally as close as possible between adhesive and bracket until debonding $(6,18)$. The actual debonding strengths were calculated as described by Bishara and recorded in megapascals $(\mathrm{MPa})(1,18)$ (Fig. 1). After debonding, the remaining adhesive was removed by an 1172-carbide bur at $30000 \mathrm{rpm}$.

Two expert orthodontists calculated the crack lengths and number through observation under optical stereomicroscope (Olympus, SZX 9) at $\times 10$ magnifications and trans-illumination technique using a ruler in the center of the lens before bonding and after debonding. Then they were registered by digital photography. (Fig. 2) Kappa coefficient of 0/78 (good agreement) was obtained between two observers.

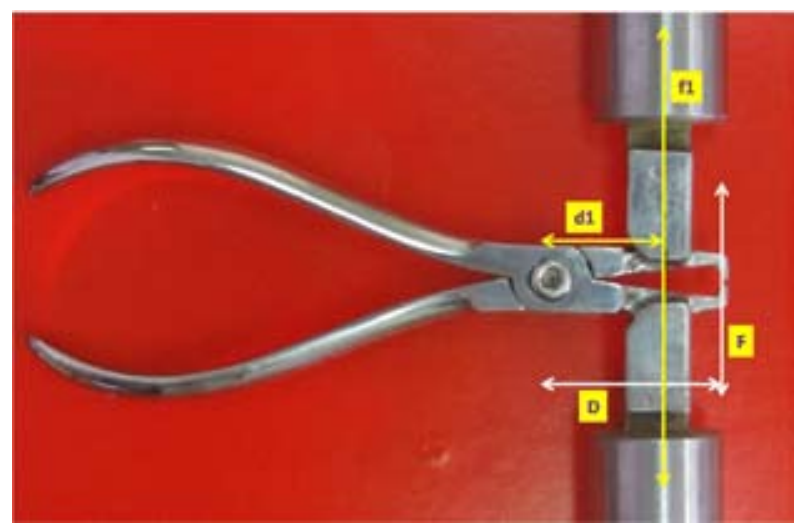

$F=$ actual debonding force

$f l=$ force applied by testing machine

$D=$ the distance from pliers edge to hinge of the pliers $(20 \mathrm{~mm})$

$d I=$ the distance from midpoint of soldered metal bar to hinge of the pliers $(15 \mathrm{~mm})$

Fig. 1. Designed of vertically oriented force system.

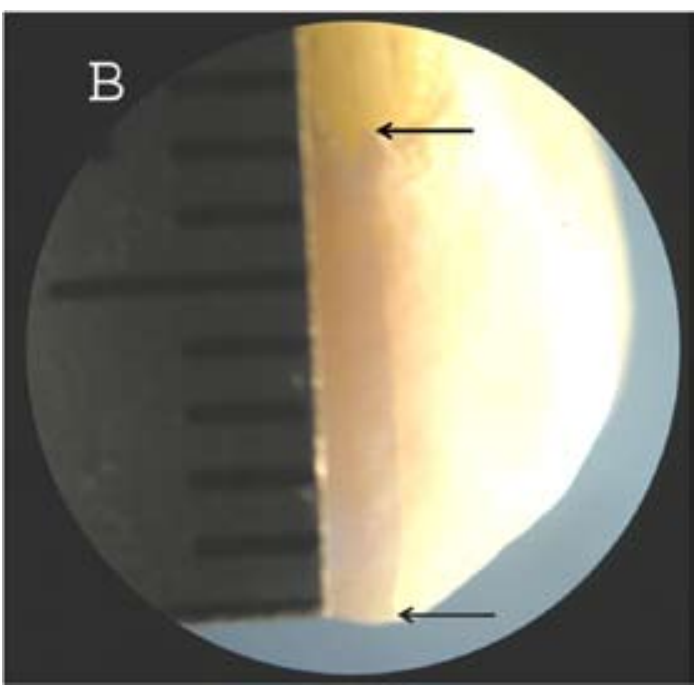

Fig. 2. Enamel crack after debonding.
The statistic analysis was performed using SPSS 13. One-Sample Kolmogorov-Smirnov Test showed the normal distribution of the data for the variables of crack length and bonding strength so the parametric analysis was used.

Paired t-test analysis was used to compare crack length before bonding and after debonding in groups. Inter group comparison was performed using Independent ttest analysis for mean bonding strength and crack lengths. McNemar's test was used to compare the number of samples with crack before bonding and after debonding both in $\mathrm{CB}$ and $\mathrm{MB}$ groups.

\section{Results}

Mean and Standard deviation of the actual debonding force were obtained $6.75 \pm 1.26 \mathrm{MPa}$ for mechanicallybonded ceramic brackets and $9.02 \pm 1.73 \mathrm{MPa}$ for metallic brackets which according to independent t-test the difference was significant statistically. $(\mathrm{P}=0.0001)$

The mean crack length was increased from $0.37 \pm$ $0.78 \mathrm{~mm}$ before bonding to $1.67 \pm 1.88 \mathrm{~mm}$ after debonding in $\mathrm{MB}$ group and from $0.30 \pm 0.69 \mathrm{~mm}$ before bonding to $1.60 \pm 1.65 \mathrm{~mm}$ after debonding in $\mathrm{CB}$ group which according to the paired t-test the differences were significant $(\mathrm{P}=0.0001)$.

The number of the teeth with enamel cracks was increased from $0.23 \%$ before bonding to $53,3 \%$ after debonding in MB group and from $0.20 \%$ before bonding to $56.7 \%$ after debonding in $\mathrm{CB}$ group which according to the McNemar's test, the differences were significant $(\mathrm{P}=$ 0.007 for $\mathrm{MB}$ group and $\mathrm{P}=0.024$ for $\mathrm{CB}$ group).

Independent t-test sowed no statistically significant differences between metallic and ceramic bracket groups with respect to crack length. $(\mathrm{P}>0.05)$

\section{Discussion}

According to some studies, although high bonding strength of adhesive is required to prevent bracket detachment during orthodontic treatment, this should be optimum in order to prevent permanent damages to the enamel at debonding $(8-11,14,19)$. The amount of bonding strength in different studies has been reported in the range between 5 to $25 \mathrm{MPa}$ and the recommended range was 5.1 to $9 \mathrm{MPa}$. It has been reported that bonding strength more than $13.5 \mathrm{MPa}$ could cause enamel fracture during debonding specially in cases when the fracture site is in the interface between adhesive and enamel (20). In this study the amount of actual debonding force for mechanically-bonded ceramic brackets and metallic brackets was $6.75 \pm 1.26 \mathrm{MPa}$ and $9.02 \pm 1.73 \mathrm{MPa}$ respectively which could be considered in the recommended range. The use of thermo-cycling in our study might have been the reason for lesser bonding strength found in this study compared to the study of Habibi et al. (18). In this study, metallic brackets demonstrated signifi- 
cantly higher force to debond compared to mechanicallybonded ceramic brackets $(\mathrm{P}=0.0001)$. This finding is in accordance with the study by Fernandez and Canut (9) and Habibi et al (18) but is not consistent with the results of studies carried out by Bishara et al (16) and Liu et al (21).

Although some studies report relatively high enamel damage subsequent to debonding ceramic brackets with chemical bonding compared to metallic brackets with mechanical retention $(5,14)$, others did not confirm this issue $(22,23)$.

In the study by Tehranchi et al (24), deboning ceramic brackets with chemical retention using super pulse $\mathrm{CO} 2$ laser showed a significant reduction of enamel damage. Though studies on debonding metallic bracket confirm damages on enamel followed by debonding they did not report any significant differences among techniques of debonding or adhesives used for bonding $(25,26)$

Recently, new ceramic brackets with notches and undercuts in the base have been introduced which provide mechanical retention (9). Some studies did not demonstrated any permanent damage to tooth enamel after debonding of ceramic brackets with mechanical retention $(8,15)$ while others report enamel damages subsequent to debonding $(9,10,18,27)$. Differences in the results of studies might be attributed to different retention mechanisms of brackets, the method of bonding and the type of adhesive.

It has been reported that bonding strength more than 13.5 $\mathrm{MPa}$ could cause enamel fracture during debonding, especially in cases when the fracture site is in the interface between adhesive and enamel (28). In this study, in spite of less force to debond strength obtained for the groups there was an statistically significant increase in the number of teeth with cracks and the length of cracks after debonding in both metallic and ceramic brackets $(p<0.001)$. The same result was obtained by the study of Shahabi et al (29) Also there were no significant differences in the number of teeth with cracks and crack length between two kinds of brackets after debonding. These findings were parallel to results obtained by Bishara et al $(1,28)$, Arici et al $(20)$ and Habibi et al (18) but were not consistent with the studies of Fernandez and Canut (9) and Blalock and Powers (4). The different results might be attributed to the kind of force application (one way force or use of pliers) and the kind of bonding mechanism for ceramic brackets (chemically or mechanically bonding). Habibi et al (18) did not find any significant differences in crack lengths between metal brackets and ceramic brackets or between ceramic brackets with mechanical retention and ceramic brackets with chemically retention. Our study, with more samples used for each group, confirmed these findings.

In our study $56 / 7 \%$ of the teeth bonded with ceramic brackets with mechanical retention and $53,3 \%$ of the teeth bonded with metallic brackets showed cracks after debonding. These findings are much higher than those obtained by other studies $(5,18,30)$. The increased number of the cracks in our study compared to previous studies might be attributed to the difference in the technique used for debonding (use of players instead of Instron machine).

In this study bracket base fitness and thermo cycling were carefully monitored. Unlike some previous studies that used one way directed cutting force, a particular method of debonding was designed bracket removal was done using a diametric compressive force from doubleedged using a pair of double edged debonding pliers that was capable of applying a true compressive force. Thus method of study was very similar to what is used in clinical situations. This method has been accepted safe and effective for debonding metallic brackets and ceramic brackets with mechanical retention as well (1, $5,22,23)$.

As a conclusion, the possible risk of producing enamel cracks after debonding in ceramic and metallic brackets both with mechanical retention, should be considered in the evaluation of benefit/risk ratio of the patients" treatment.

For bonding brackets it is recommended to use techniques and adhesives in acceptable range of bonding strength to prevent enamel damages after debonding and the patient should be informed about possible risk of enamel damages apart from the type of bracket used.

This study may encourage further studies on the deepness of cracks caused by debonding.

\section{Acknowledgments}

The authors would like to thank the research foundation of Tabriz University of Medical Sciences for the financial support of the study.

\section{References}

1. Bishara SE, Forrseca JM, Fehr DE, Boyer DB. Debonding forces applied to ceramic brackets simulating clinical conditions. Angle Orthod. 1994;64:277-82.

2. Winchester LJ. Bond strengths of five different ceramic brackets: an in vitro study. Eur J Orthod. 1991;13:293-305.

3. Idiyatullin D, Corum C, Moeller S, Prasad HS, Garwood M, Nixdorf DR. Dental magnetic resonance imaging: making the invisible visible. J Endod. 2011;37:745-52.Epub 2011 Apr 6.

4. Blalock KA, Powers JM. Retention capacity of the bracket bases of new esthetic orthodontic brackets. Am J Orthod Dentofacial Orthop. 1995;107:596-603.

5. Bishara SE, Fonseca JM, Boyer DB. The use of debonding pliers in the removal of ceramic brackets: force levels and enamel cracks. Am J Orthod Dentofacial Orthop. 1995;108:242-8.

6. Wang WN, Meng CL, Tarng TH. Bond strength: a comparison between chemical coated and mechanical interlock bases of ceramic and metal brackets. Am J Orthod Dentofacial Orthop. 1997;111:374-81.

7. Bishara SE, Ajlouni R, Laffoon JF. Effect of thermocycling on the shear bond strength of a cyanoacrylate orthodontic adhesive. Am J Orthod Dentofacial Orthop. 2003;123:21-4. 
8. Kapur R, Sinha PK, Nanda RS. Comparison of frictional resistance in titanium and stainless steel brackets. Am J Orthod Dentofac Orthop. Am J Orthod Dentofacial Orthop. 1999;116:271-4.

9. Bishara SE, Fehr DE. Ceramic brackets: something old, something new -a review, Semin Orthod. 1997;3:178.

10. Olsen M, Bishara S, Boyer D, Jakobson J. Effect of varying etching times on the bond strength of ceramic brackets. Am J Orthod Dentofac Orthop. 1996;109:403-9.

11. Bishara SE. Ceramic brackets and the need to develop national standards. Am J Orthod Dentofac Orthop. 2000;117:595-7.

12. Eliades T, Brantley W. The inappropriateness of conventional orthodontic bond strength assessment protocols. Eur J Orthod. 2000;22:13-23.

13. Fernandez L, Canut J. In vitro comparison of the retention capacity of new aesthetic brackets. Eur J Orthod. 1999;21:71-7.

14. van Waveren Hogervorst WL, Feilzer AJ, Prahl-Andersen B. The air-abrasion technique versus the conventional acid-etching technique: A quantification of surface enamel loss and a comparison of shear bond strength. Am J Orthod Dentofacial Orthop. 2000;117:20-6.

15. Ajlouni R, Bishara SE, Oonsombat C, Soliman M, Laffoon J. The effect of porcelain surface conditioning on bonding orthodontic brackets Angle Orthod. 2005;75:858-64.

16. Bishara SE, Oonsombat C, Soliman MM, Warren JJ, Laffoon JF, Ajlouni R. Comparison of bonding time and shear bond strength between a conventional and a new integrated bonding system. Angle Orthod. 2005;75:237-42.

17. Arnold RW, Combe EC, Warford JH Jr. Bonding of stainless steel brackets to enamel with a new self-etching primer. Am J Orthod Dentofacial Orthop. 2002;122:274-6.

18. Habibi M, Nik TH, Hooshmand T. Comparison of debonding characteristics of metal and ceramic orthodontic brackets to enamel: an in-vitro study. Am J Orthod Dentofacial Orthop. 2007;132:675-9.

19. Olsen ME, Bishara SE, Damon P, Jakobsen JR. Evaluation of Scotchbond Multipurpose and maleic acid as alternative methods of bonding orthodontic brackets. Am J Orthod Dentofacial Orthop. 1997;111:498-501.

20. Arici S, Minors C. The force levels required to mechanically debond ceramic brackets. Eur J Orthod. 2000;22:327-34.

21. Liu JK, Chung CH, Chang CY, Shieh DB. Bond strength and debonding characteristics of a new ceramic bracket. Am J Orthod Dentofacial Orthop. 2005;128:761-5.

22. Chen HY, Su MZ, Chang HF, Chen YJ, Lan WH, Lin CP. Effects of different debonding techniques on the debonding forces and failure modes of ceramic brackets in simulated clinical set-ups. Am J Orthod Dentofacial Orthop. 2007;132:680-6.

23. Bishara SE, Ostby AW, Laffoon J, Warren JJ. Enamel cracks and ceramic bracket failure during debonding in vitro. Angle Orthod. 2008;78:1078-83.

24. Tehranchi A, Fekrazad R, Zafar M, Eslami B, Kalhori KA, Gutknecht $\mathrm{N}$. Evaluation of the effects of $\mathrm{CO} 2$ laser on debonding of orthodontics porcelain brackets vs. the conventional method. Lasers Med Sci. 2011;26:563-7

25. Heravi F, Rashed R, Raziee L. The effects of bracket removal on enamel. Aust Orthod J. 2008;24:110-5.

26. Tecco S, Tetè S, D'Attilio M, Festa F. Enamel surface after debracketing of orthodontic brackets bonded with flowable orthodontic composite. A comparison with a traditional orthodontic composite resin. Minerva Stomatol. 2008;57:81-94.

27. Sinha PK, Nanda RS. The effect of different bonding and debonding techniques on debonding ceramic orthodontic brackets. Am J Orthod Dentofacial Orthop. 1997;112:132-7

28. Bishara SE, Fehr DE, Jakobsen JR. A comparative study of different ceramic brackets enamels conditioners and adhesives. Am J Orthod Dentofacial Orthop. 1993;104:170-9.

29. Shahabi M, Heravi F, Mokhber N, Karamad R, Bishara SE. Effects on shear bond strength and the enamel surface with an enamel bonding agent. Am J Orthod Dentofacial Orthop. 2010;137:375-8.

30. Lee RT. How to ... debond Clarity brackets with ease. J Orthod.
2005;32:269-71. 